OPEN ACCESS

Edited by:

Shou-Long Deng,

Chinese Academy of Medical

Sciences and Peking Union Medical

College, China

Reviewed by:

Guozhi Yu,

Sichuan Agricultural University, China

Shanliang $\mathrm{Li}$,

Guangxi University of Chinese

Medicine, China

${ }^{*}$ Correspondence:

Weizheng Liang

LYjm210115@163.com

Chengwei Yu

yuchengwei2019d@big.ac.cn

Hao Wang

earthminator@sina.cn

Hui Liu

jivi111@126.com

${ }^{\dagger}$ These authors have contributed equally to this work and share first authorship

Specialty section:

This article was submitted to

Cell Death and Survival,

a section of the journal

Frontiers in Cell and Developmental

Biology

Received: 09 September 2021

Accepted: 08 November 2021

Published: 16 December 2021

Citation:

Liang W, Liu H, He J, Ai L, Meng Q, Zhang $W, Y u C$, Wang $H$ and Liu $H$

(2021) Studies Progression on the

Function of Autophagy in

Viral Infection.

Front. Cell Dev. Biol. 9:772965.

doi: 10.3389/fcell.2021.772965

\section{Studies Progression on the Function of Autophagy in Viral Infection}

\author{
Weizheng Liang ${ }^{1 \star t}$, Huimin $\mathrm{Liu}^{2 \dagger}$, Junli He${ }^{3}$, Lisha $\mathrm{Ai}^{4}$, Qingxue Meng ${ }^{5}$, Weiwen Zhang ${ }^{6}$, \\ Chengwei $\mathrm{Yu}^{7,8 *}$, Hao Wang ${ }^{5,6_{*}}$ and Hui Liu ${ }^{9 *}$ \\ ${ }^{1}$ Harbin Institute of Technology, Harbin, China, ${ }^{2}$ Department of Infectious Diseases, Southwest Hospital, Third Military Medical \\ University (Army Medical University), Chongqing, China, ${ }^{3}$ Department of Pediatrics, Shenzhen University General Hospital, \\ Shenzhen, China, ${ }^{4}$ Department of Teaching and Research, Shenzhen University General Hospital, Shenzhen, China, ${ }^{5}$ Department \\ of Science, Southern University of Science and Technology, Shenzhen, China, ${ }^{6}$ Department of Gynaecology and Obstetrics, \\ Shenzhen University General Hospital, Shenzhen, China, ${ }^{7}$ School of Future Technology, University of Chinese Academy of \\ Sciences, Beijing, China, ${ }^{8}$ CAS Key Laboratory of Genome Sciences and Information, Beijing Institute of Genomics, Chinese \\ Academy of Sciences, Beijing, China, ${ }^{9}$ Department of Hepatobiliary Surgery, Shenzhen University General Hospital, Shenzhen, \\ China
}

Autophagy is a conservative lysosomal catabolic pathway commonly seen in eukaryotic cells. It breaks down proteins and organelles by forming a two-layer membrane structure of autophagosomes and circulating substances and maintaining homeostasis. Autophagy can play a dual role in viral infection and serve either as a pro-viral factor or an antiviral defense element dependent on the virus replication cycle. Recent studies have suggested the complicated and multidirectional role of autophagy in the process of virus infection. On the one hand, autophagy can orchestrate immunity to curtail infection. On the other hand, some viruses have evolved strategies to evade autophagy degradation, facilitating their replication. In this review, we summarize recent progress of the interaction between autophagy and viral infection. Furthermore, we highlight the link between autophagy and SARS-CoV-2, which is expected to guide the development of effective antiviral treatments against infectious diseases.

Keywords: autophagy, coronavirus, virus, infection, SARS-CoV-2

\section{INTRODUCTION}

Autophagy is an evolutionary conserved lysosome-dependent cellular metabolic pathway in eukaryotic cells by degrading senescent organelles and long-lived proteins into small molecules such as peptides or amino acids through which the material recycling and intracellular homeostasis can be maintained (Chiang, Terlecky et al., 1989; Rubinsztein, Marino et al., 2011; Wei, Liu et al., 2018; Levine and Kroemer 2019). Meanwhile, autophagy has critical functions in cell growth and development (Levine, Mizushima et al., 2011; Rubinsztein, Marino et al., 2011; Mizushima 2018). The existing studies have demonstrated that various diseases can cause autophagy dysfunction, including tumors, neurodegenerative diseases, cardiovascular diseases, and viral infections (Levine and Kroemer 2008; Mizushima 2018; Mizushima and Levine 2020). As part of the human body's protective and defensive mechanisms, autophagy is essential in the face of pathogenic microbial invasion, whereby cells can attack the "invaders" through autophagic degradation. However, lots of pathogenic microorganisms, especially viruses, have evolved various strategies to evade host cell autophagy and infect the organism persistently, and some viruses can even use the autophagic machinery to promote their replication in the host (Choi, Bowman et al., 2018; Yang and Klionsky 2020). 


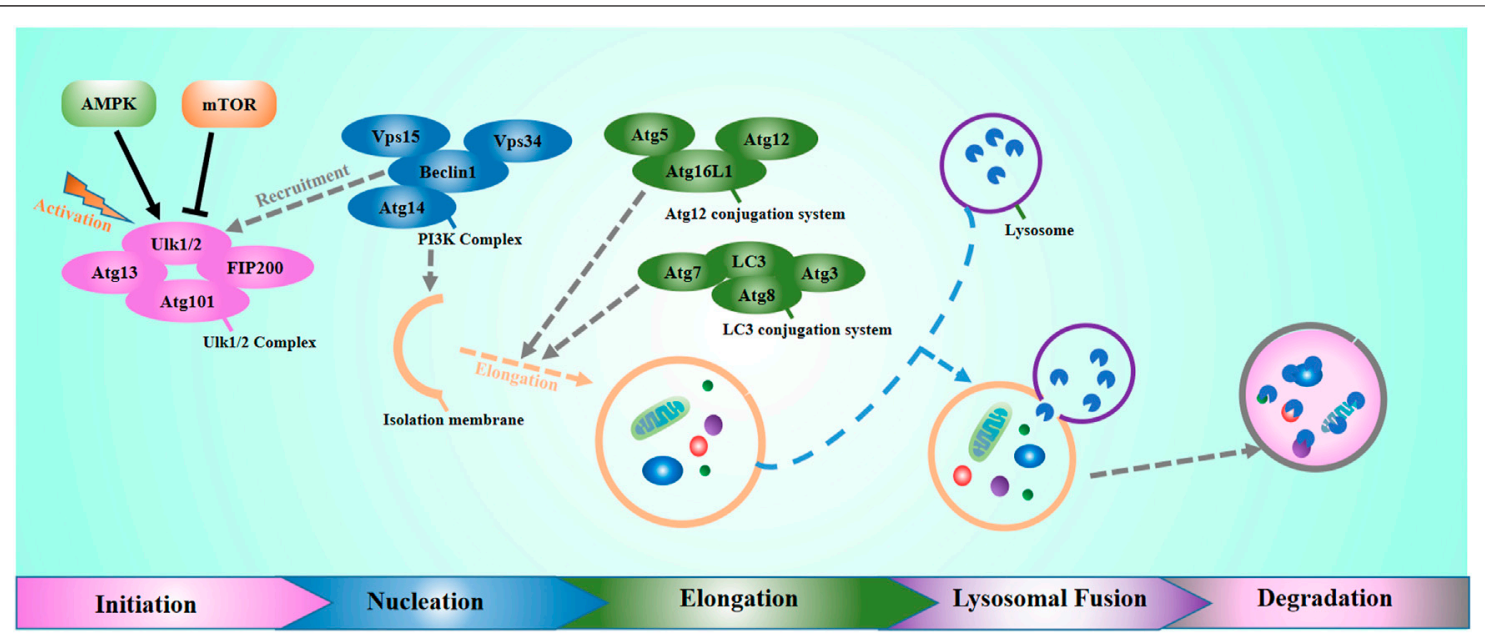

FIGURE 1 | The molecular process of autophagy, including initiation, nucleation, elongation, lysosomal fusion, and degradation.

The functions of cellular autophagy in viral infection have been studied elsewhere. This review focuses on a detailed portrait of the connection between autophagy and viral infections in cells. It could be useful to understand viral infection diseases and find proper medical strategies to treat them.

\section{CLASSIFICATION AND PROCESS OF AUTOPHAGY}

Autophagy is a ubiquitous biological mechanism in eukaryotes that contributes to maintaining homeostasis in the organism. Generally speaking, autophagy includes three main types: microautophagy, molecular chaperone-mediated autophagy, and macroautophagy (Oku and Sakai 2018). This review focuses on macroautophagy, which is the most important autophagy form. In normal physiological conditions, the intracellular autophagy level is at the basal level. The intracellular or extracellular stress can strongly bring up autophagy levels to maintain cellular homeostasis. Until now, more than 30 genes have been found to participate in the regulation of autophagy (Choi, Ryter et al., 2013; Saha, Panigrahi et al., 2018). Those genes are autophagy-related genes. In mammals, the classical autophagic pathway has five stages: initiation, nucleation, elongation, lysosomal fusion, and degradation of autophagic contents (Figure 1). Autophagy initiates at the activation of the Unc-51 like autophagy activating kinase 1 (ULK1) complex. ULK1 is a mammalian homolog of autophagy-related gene 1 (ATG1), which interacts with the adherent plaque kinase family (FAK family), the autophagy-related gene Atg13, and Atg101 to form the ULK1 complex (Zachari and Ganley 2017). ULK1 activation promotes the recruitment of multiprotein complexes with class III phosphoinositide 3-kinase (PI3K-III, also known as PIK3C3) activity. Beclin-1 is a direct downstream target of ULK1, which regulates the lipid kinase activity of the PI3K-III catalytic unit vacuolar protein sorting34 (Vps34). Vps34 produces phosphatidylinositol 3-phosphate [PI (3-phosphate)], which enables the recruitment of many autophagic proteins involved in autophagosome nucleation. When autophagy is induced, the Vps34-Vps15-Beclin-1 complex can bind with different autophagy-related proteins, delivering autophagic signals, and promoting autophagy (McKnight and Zhenyu 2013). During the nucleation phase, the bilayer membrane structure of the phagophore wrap the molecules to be degraded to form a sequestering compartment. In this phase, the p62 acts as a bridging protein in the autophagy process, bringing the reduant. dysfunctional and damaged cellular components, like excess mitochondria and unfolded proteins to the phagophore, and then binds with microtubuleassociated protein 1 light chain 3 [protein light chain3 (LC3)] (Chen, Lam et al., 2010; Tanaka, Jin et al., 2012; Ge, Wilz et al., 2015), which traps these cargo to be degradeds in the phagophore. The partition membrane continues to bend, elongate, and completely wraps around the cytoplasm to form a complete double-membrane autophagosome. Autophagosomes can fuse with late endosomes to form amphisomes and finally form autolysosomes by fusing with lysosome. Besides, autophagosomes also can directly fuse with lysosomes to form autolysosomes.

\section{THE REGULATORY FUNCTIONS OF AUTOPHAGY IN VIRAL INFECTION}

It has been previously reported that autophagy is not only a metabolic pathway in the organism to maintain homeostasis, but also it can effectively play antiviral functions as an irreplaceable component of the human immune defense system. After the virus invades into cells, the viral proteins, nucleic acids, and viral particles can be brought into the lysosome for degradation through autophagic signaling pathway, thus maintaining the health of the organisms (Levine 2005; Levine and Kroemer 2019; Yang and Klionsky 2020). Viruses are intracellular 
parasitic microorganisms with only one nucleic acid type. Autophagy may be triggered by any step in the life cycle of viruses, including viral adsorption and invasion nucleic acid replication and protein synthesis, and so on (Lünemann and Münz 2009; Tey and Khanna 2012; Perot, Ingersoll et al., 2013). Autophagy has antiviral or viral promotion functions during viral infection, depending on the virus, cell type, and cell environment (Jackson 2015; Pleet, Branscome et al., 2018). At present, it has been found that virus invasion induces autophagy in three ways. 1). Virus proteins can directly induce autophagy (Gannagé, Dormann et al., 2009; Londino, Lazrak et al., 2015). 2) Autophagy can be induced by virus ligand binding to the receptor (Joubert, Meiffren et al., 2009; Richetta, Grégoire et al., 2013). 3) Viruses-related endoplasmic reticulum stress induces autophagy. Viruses can encode a large number of viral proteins in infected cells. However, host cells cannot effectively modify these proteins in the endoplasmic reticulum. Those proteins cannot be correctly folded, resulting in protein accumulation in the endoplasmic reticulum, triggering endoplasmic reticulum stress, and activating autophagy in cells.

Autophagy can transport the virus in the cytoplasm to the lysosome by heterologous phagocytosis, directly degrade the virus, and transport viral pathogen-associated molecular patterns (PAMPs) to the intracellular receptors to activate the innate immunity. It can also present the virus antigen to the major histocompatibility complex (MHC) to activate the adaptive immunity and then trigger the function of resisting virus infection.

It is well known that innate immunity is the organism's firstline defense against invasion by exogenous microorganisms. The PRRs (pattern recognition receptors) recognize and bind to specific molecular structures of pathogenic microorganisms and activate downstream signaling pathways to produce type I interferon (IFN), type III IFN, pro-inflammatory factors, and chemokines to establish the antiviral state (Kawai and Akira 2010; Stanifer, Pervolaraki et al., 2019).

Toll-like receptor (TLR) is a classical PRR that recognizes multiple viral nucleic acids. By recruiting adaptor molecule 1 (TRIF) or myeloid differentiation factor MyD88, the signal can be transmitted to the downstream NF- $\kappa \mathrm{B}$ signaling pathway and synthesizes inflammatory factors and IFN regulatory factors (IRFs), promoting IFN production (Lee and Kim 2007; Negishi, Taniguchi et al., 2018). In this process, TLR can induce the interaction between TRIF or MyD88 and Beclin 1, by which the binding between Beclin 1 and Bcl-2 can thus induce autophagy (Zalckvar, Berissi et al., 2009; Kang, Zeh et al., 2011). On the other hand, autophagy can also be directly activated by pathogen-associated molecular patterns (PAMP) and transmit PAMP signals to TLRs to induce immune responses (Chen, Yang et al., 2012). Song J et al. found in human bronchial epithelial cells (16HBE) that autophagy could induce the increased replication of EV71 and CA16 (Song, Hu et al., 2018). Therefore, during the infection of a few viruses, autophagy can negatively regulate the TLR signaling pathway, thus contributing to the self-proliferation of the virus.

RIG-I-like receptors (RLRs) are localized in the cytoplasm and are mainly responsible for recognizing specific structures that are not present in normal cellular RNAs, such as the $5^{\prime}$ end triphosphate of viral dsRNA (Chiang, Davis et al., 2014). The RLRs sense the presence of the virus and interact with mitochondrial antiviral signals (MAVS) through the caspase recruitment domain (CARD), which in turn activates the regulatory factors IRF3 and IRF7 and NF- $\kappa$ B signaling pathways (Chiang, Davis et al., 2014). It was found that Sendai virus-infected cells could induce autophagy, but not in RIG-I-deficient cells (Lee, Ban et al., 2018), suggesting that RIG-I plays a role in virus-induced autophagy. The function of RIG-I is related to its molecular stability. In addition to ubiquitinproteasome degradation, it also includes autophagic pathways. Leucine-rich Recombinant Protein 25 (LRRC25) can act as a secondary receptor to facilitate the recognition of ISG15-related RIG-I by SQSTM1/p62, mediating the degradation of RIG-I through selective autophagy (Du, Duan et al., 2018; Xu, Wan et al., 2018).

In addition, a few receptor molecules that sense DNA virus invasion in cells can also interact with the autophagic pathway. Cyclic GMP-AMP synthase (cGAS) can synthesize cyclic GMP -AMP (Cyclic GMP -AMP) after recognizing DNA viruses, followed by the STING factor binding to cGAMP to activate downstream related regulators and induce NF-kB activation. Among them, ERGIC containing STING can provide a membrane source for LC3 lipidation, thus promoting the clearance of cytoplasmic virus DNA by autophagy (Gui, Yang et al., 2019). On the other hand, during HSV-1 infection, Beclin 1 interacts with cGAS to inhibit cGAMP synthesis and IFN production and enhances autophagy-mediated degradation of cytoplasmic pathogen DNA to prevent excessive cGAS activation and sustained immune stimulation (Liang, Seo et al., 2014; Ni, Ma et al., 2020). Therefore, autophagy or autophagy-related proteins are not only regulated by signals such as cGAS-STING. However, they can also negatively regulate related signaling pathways.

Delivery of antigen is a transitional process during which natural immunity is the shift to adaptive immunity. In this process, antigen peptide fragments activate the major histocompatibility complex (MHC) in antigen-presenting cells (APCs), followed by antigen recognition by $\mathrm{T}$ cells in organisms. MHC mainly consists of two categories: MHC-I and MHC-II. MHC-I can be expressed in all nucleated cells. The viral protein can be delivered to $\mathrm{CD} 8^{+} \mathrm{T}$ cells through MHC-I-like molecules after degradation by the proteasome into immunogenic peptides, while autophagy limits MHC-I-like molecules by mediating their internalization and degradation (Choi, Bowman et al., 2018). The inactivation of the autophagy factors ATG5 and ATG7 in dendritic cells (DCs) can increase the expression of MHC-I on the cell surface, which makes the body induce a stronger immune response when infected with IAV and LCMV (Loi, Müller et al., 2016).

On the other hand, autophagosomes form constitutively in MHC-class II positive APCs such as dendritic cells, B cells, and epithelial cells, and APCs capture extracellular antigens and deliver them to autophagosomes. The autophagosomes fuse with MHC-II containing late nuclear endosomal compartments (MIIC) to deliver cytoplasmic antigen into MHC-II-like molecules, then delivered to $\mathrm{CD}^{+} \mathrm{T}$ cells 
(Dengjel, Schoor et al., 2005). Studies have shown that after HIV infection into DCs, cells can utilize LC3 fusion protein to target HIV antigens to autophagosomes specifically. In this way, the immune response of $\mathrm{CD}^{+} \mathrm{T}$ cells is effectively enhanced and amplified, thereby facilitating the restricted expression of MHCII (Coulon, Richetta et al., 2016). In addition, autophagy can mediate the cross-antigen presentation of MHC-I and MHC-II types in APCs, facilitating information exchange between cells to generate effective immune responses against endogenous and exogenous antigens (English, Chemali et al., 2009). Autophagy also contributes to the developmental maturation of immune cells. The deficiency of autophagy-related genes, ATG5 or ATG7, in $\mathrm{T}$ cells can decrease the number of thymocytes and peripheral T cells. Besides, in ATG5-deficient T cells, the accumulation of damaged and aged mitochondria can cause cell proliferation problems and reduced cell viability (Stephenson, Miller et al., 2009). The mice with ATG7-deficient T cells fail to effectively establish $\mathrm{CD}^{+} \mathrm{T}$ cell responses to influenza and murine cytomegalovirus (MCMV) infection, suggesting that autophagy is essential for the survival of effector $\mathrm{CD}^{+} \mathrm{T}$ cells and immune memory formation (Puleston, Zhang et al., 2014; Xu, Araki et al., 2014).

\section{THE SPECIFIC FUNCTIONS OF SEVERAL CLASSICAL VIRUSES}

During the host infection, autophagy can degrade the virus and promote the innate and adaptive immunity of the host to inhibit viruses. However, it has been known that quite a few adaptive viruses have evolved a variety of biological mechanisms to regulate autophagy in the process of long-term co-existence with the host, such as using autophagy to inhibit cellular innate immunity to escape immune surveillance or by directly inhibiting the formation of autophagosomes, blocking the fusion of autophagosomes and lysosomes, and even using autophagosomes as a place for their replication to achieve the purpose of survival and reproduction. Studies have found that different viruses and different stages of infection have different strategies for regulating autophagy (Sir and Ou 2010; Abdoli, Alirezaei et al., 2018; Huang, Liu et al., 2020). The functions of autophagy in several viruses are described below.

Previous studies showed that $\mathrm{x}$ protein overexpression in hepatitis B virus (HBV) could significantly up-regulate Beclin 1 and promote autophagy levels in liver cancer cells (Tang, Da et al., 2009). Nevertheless, another study indicated that, by triggering endoplasmic reticulum stress, the small surface protein SHBs of HBV can effectively activate autophagy, and SHBs protein and autophagy protein LC3 co-localize and interact during HBV replication. The application of autophagy inhibitor 3-methyladenine (3-MA) to inhibit autophagy can significantly inhibit HBV production, and the application of rapamycin or starvation to induce autophagy can promote the production of $\mathrm{HBV}$. The results suggested that autophagy was involved in HBV replication (Li, Liu et al., 2011).

Hepatitis $\mathrm{C}$ virus (HCV) is a single-stranded (+) RNA virus. Studies have shown that this HCV is also associated with autophagy. Its function may have two sides: HCV invading cells can cause endoplasmic reticulum stress, increasing autophagosome. However, the fusion of autophagosomes and lysosomes is inhibited. This incomplete autophagy mechanism allows HCV to use the autophagosomal membrane to replicate viral RNA, thus facilitating HCV replication (Shrivastava, Raychoudhuri et al., 2011). In contrast to this finding, other studies have found that the IFNbeta-induced endoplasmic reticulum transmembrane protein SCOTIN can recruit the NS5A protein from $\mathrm{HCV}$ to the autophagosome for degradation (Kim, Kim et al., 2016). In addition, IFN- $\lambda 1$ can suppress the expression of ATG5 and GABARAP by inducing the expression of miR-181a and miR-214, thereby downregulating autophagy to inhibit HCV replication (Li, Li et al., 2017).

Avian influenza virus $\mathrm{H} 5 \mathrm{~N} 1$ infection can induce cells to produce inflammatory cytokines leading to body damage by producing ROS. H5N1 infection of A549 lung epithelial cells can induce the formation of obvious autophagosomes. Applying autophagy inhibitor 3-MA or knockdown of autophagy gene Atg5 by siRNA can alleviate H5N1-induced acute lung injury. Further studies have found that $\mathrm{H} 5 \mathrm{~N} 1$ can mediate lung injury by inhibiting Akt/TSC2/mTOR pathway to activate autophagy (Imai, Kuba et al., 2008). Therefore, targeting the autophagy pathway could be a promising method for the medical treatment of lung inflammation caused by the H5N1 virus.

The human immunodeficiency virus (HIV) can manipulate the autophagy process by interacting with different autophagy factors. In the early stage of autophagy, HIV Gag protein binds and interacts with LC3, thereby promoting the processing of Gag protein and the maturation and packaging of viral particles for HIV replication. When autophagy enters the mature stage, HIV Nef protein plays an anti-autophagy maturation role by interacting with autophagy protein Beclin-1, thereby protecting HIV from degradation and promoting the release of mature virus particles. Therefore, HIV can promote HIV survival and replication by interfering with different stages of autophagy (Kyei, Dinkins et al., 2009; Leymarie, Lepont et al., 2017).

Human Bocavirus 1 (HBoV1) is a parvovirus, which can cause serious respiratory diseases in children. NP1 protein of HBoV1 can up-regulate Beclin1 and LC3II in A549 cells but downregulate the expression of p62 and HMGB1 and inhibit cell survival and migration. In addition, it is also found that the STAT3 signaling pathway activates NP1-induced autophagy in A549 cells. The above results show that NP1 of HBoV1 induces autophagy in A549 cells by activating STAT3 signaling pathway phosphorylation and inhibits the viability of A549 cells. However, the mechanism of NP1-induced autophagy remains unclear (Kim, Lee et al., 2010; Lennemann and Coyne 2015; Mancias and Kimmelman 2016; Padman, Nguyen et al., 2017).

\section{AUTOPHAGY AND SARS-COV-2}

A new coronavirus was discovered in late 2019 (Severe acute respiratory syndrome coronavirus 2 . SARS-CoV-2), which spread rapidly in a short period and attracted worldwide attention. With the COVID-19 pandemic caused by the SARS-Cov-2, as of 
September 9, 2021, more than 222,136,320 cases were reported worldwide, including 4,590,565 death reports (Data from coronavirus resource center of Johns Hopkins University). Due to the epidemic's severity, SARS-CoV-2 is currently one of the most important viruses in the academic community. Developing a specific drug or vaccine for SARS-CoV-2 has become a hot research topic (Weston, Coleman et al., 2020). Combing with the findings on other coronaviruses and the previous clinical survey showed that lots of medicines approved by the US Food and Drug Administration (FDA) can effectively regulate autophagic pathway (Bello-Perez, Sola et al., 2020; Shojaei, Suresh et al., 2020). This viewpoint suggests that the accumulation of autophagsosomes caused by interfering autophagic pathway may be a potential target for controlling SARS-CoV-2 by inducing apoptosis of infected cells. As proof of concept, both Chloroquine (CQ) and Hydroxychloroquine (HCQ) (HCQ) are two of the drugs of interest for clinical evaluation (Elavarasi, Prasad et al., 2020; Kumar, Jain et al., 2021; Singh, Ryan et al., 2021). They can inhibit the autophagic process by increasing the $\mathrm{pH}$ of acidic endosomes/lysosomes. In contrast to this point, other study found that through inhibiting the function of autophagy resisting pathogens, SARS-CoV-2 can survive and replicate in cells without protection. Therefore, increasing the autophagy flux level is also the way to treat SARS-CoV-2 (Miao, Zhao et al., 2021). Hannan et al. mentioned that intermittent fasting could be a potential approach to treat the infection SARSCoV-2 by the activation of autophagy (Hannan, Rahman et al., 2020). These opinions suggest that autophagy regulation is indispensable in the fighting against SARS-CoV-2 infection.

\section{SUMMARY AND PERSPECTIVES}

Autophagy, as a conservatively evolved intracellular degradation pathway, is a multi-step and complex process. Autophagy can deliver the virus PAMPs to pattern recognition receptors to activate innate immunity. Additionally, it can present viral antigens processing to MHC molecules to activate adaptive immunity in organisms. It can also transport the virus

\section{REFERENCES}

Abdoli, A., Alirezaei, M., Mehrbod, P., and Forouzanfar, F. (2018). Autophagy: The Multi-Purpose Bridge in Viral Infections and Host Cells. Rev. Med. Virol. 28 (4), e1973. doi:10.1002/rmv.1973

Bello-Perez, M., Sola, I., Novoa, B., Klionsky, D. J., and Falco, A. (2020). Canonical and Noncanonical Autophagy as Potential Targets for COVID-19. Cells 9 (7), 1619. doi:10.3390/cells9071619

Chen, G.-Y., Yang, H.-J., Lu, C.-H., Chao, Y.-C., Hwang, S.-M., Chen, C.-L., et al. (2012). Simultaneous Induction of Autophagy and Toll-like Receptor Signaling Pathways by Graphene Oxide. Biomaterials 33 (27), 6559-6569. doi:10.1016/ j.biomaterials.2012.05.064

Chen, Z.-H., Lam, H. C., Jin, Y., Kim, H.-P., Cao, J., Lee, S.-J., et al. (2010). Autophagy Protein Microtubule-Associated Protein 1 Light chain-3B (LC3B) Activates Extrinsic Apoptosis during Cigarette Smoke-Induced Emphysema. Proc. Natl. Acad. Sci. 107 (44), 18880-18885. doi:10.1073/ pnas. 1005574107 in the cytoplasm to the lysosome by heterologous phagocytosis, which plays a role in resisting virus infection. On the other hand, viruses can promote their infection by inhibiting autophagy, interacting with autophagy proteins, and even using autophagy as a replication site. Therefore, an in-depth study of the relationship between autophagy and viral infection is helpful to provide new ideas and targets for controlling viral infection.

The COVID-19 epidemic has not yet been truly resolved, and clinically effective drugs and vaccines available are still under investigation. Autophagy may be a good potential regulatory target for the treatment of the COVID-19 target. In addition, it should be noted that the regulatory function of autophagy in viral infection varies greatly depending on the virus types and the environment in which it is located. It is important to remind us to consider effectively utilizing the autophagy process as a therapeutic pathway to treat diseases caused by viruses.

\section{AUTHOR CONTRIBUTIONS}

All authors were involved in the manuscript writing. WL, HmL, and CY prepared the figure. WL, HL, HW, and CY wrote and approved the final text.

\section{FUNDING}

This work was supported by the Shenzhen Science and Technology Innovation Commission Project (Grant No. JCYJ20180302174235893), Natural Science Foundation of Guangdong Province of China (grant number 2021A1515012161), and Sanming Project of Medicine in Shenzhen (grant number SZSM202003009).

\section{ACKNOWLEDGMENTS}

We thank Prof. Chunfu Zheng for editing our manuscript.

Chiang, H.-L., Terlecky, S. R., Plant, C. P., and Dice, J. F. (1989). A Role for a 70 kilodalton Heat Shock Protein in Lysosomal Degradation of Intracellular Proteins. Science 246 (4928), 382-385. doi:10.1126/science.2799391

Chiang, J. J., Davis, M. E., and Gack, M. U. (2014). Regulation of RIG-I-like Receptor Signaling by Host and Viral Proteins. Cytokine Growth Factor. Rev. 25 (5), 491-505. doi:10.1016/j.cytogfr.2014.06.005

Choi, A. M. K., Ryter, S. W., and Levine, B. (2013). Autophagy in Human Health and Disease. N. Engl. J. Med. 368 (7), 651-662. doi:10.1056/ nejmra1205406

Choi, Y., Bowman, J. W., and Jung, J. U. (2018). Autophagy during Viral Infection a Double-Edged Sword. Nat. Rev. Microbiol. 16 (6), 341-354. doi:10.1038/ s41579-018-0003-6

Coulon, P.-G., Richetta, C., Rouers, A., Blanchet, F. P., Urrutia, A., Guerbois, M., et al. (2016). HIV-infected Dendritic Cells Present Endogenous MHC Class IIRestricted Antigens to HIV-specific CD4+ T Cells. J.I. 197 (2), 517-532. doi:10.4049/jimmunol.1600286

Dengjel, J., Schoor, O., Fischer, R., Reich, M., Kraus, M., Müller, M., et al. (2005). Autophagy Promotes MHC Class II Presentation of Peptides from Intracellular 
Source Proteins. Proc. Natl. Acad. Sci. 102 (22), 7922-7927. doi:10.1073/ pnas.0501190102

Du, Y., Duan, T., Feng, Y., Liu, Q., Lin, M., Cui, J., et al. (2018). LRRC25 Inhibits Type I IFN Signaling by Targeting ISG15-associated RIG-I for Autophagic Degradation. Embo j 37 (3), 351-366. doi:10.15252/embj.201796781

Elavarasi, A., Prasad, M., Seth, T., Sahoo, R. K., Madan, K., Nischal, N., et al. (2020). Chloroquine and Hydroxychloroquine for the Treatment of COVID-19: a Systematic Review and Meta-Analysis. J. Gen. Intern. Med. 35 (11), 3308-3314. doi:10.1007/s11606-020-06146-w

English, L., Chemali, M., and Desjardins, M. (2009). Nuclear Membrane-Derived Autophagy, a Novel Process that Participates in the Presentation of Endogenous Viral Antigens during HSV-1 Infection. Autophagy 5 (7), 1026-1029. doi:10.4161/auto.5.7.9163

Gannagé, M., Dormann, D., Albrecht, R., Dengjel, J., Torossi, T., Rämer, P. C., et al. (2009). Matrix Protein 2 of Influenza A Virus Blocks Autophagosome Fusion with Lysosomes. Cell Host \& Microbe 6 (4), 367-380. doi:10.1016/ j.chom.2009.09.005

Ge, L., Wilz, L., and Schekman, R. (2015). Biogenesis of Autophagosomal Precursors for LC3 Lipidation from the ER-Golgi Intermediate Compartment. Autophagy 11 (12), 2372-2374. doi:10.1080/ 15548627.2015.1105422

Gui, X., Yang, H., Li, T., Tan, X., Shi, P., Li, M., et al. (2019). Autophagy Induction via STING Trafficking Is a Primordial Function of the cGAS Pathway. Nature 567 (7747), 262-266. doi:10.1038/s41586-019-1006-9

Hannan, M. A., Rahman, M. A., Rahman, M. S., Sohag, A. A. M., Dash, R., Hossain, K. S., et al. (2020). Intermittent Fasting, a Possible Priming Tool for Host Defense against SARS-CoV-2 Infection: Crosstalk Among Calorie Restriction, Autophagy and Immune Response. Immunol. Lett. 226, 38-45. doi:10.1016/ j.imlet.2020.07.001

Huang, S., Liu, K., Cheng, A., Wang, M., Cui, M., Huang, J., et al. (2020). SOCS Proteins Participate in the Regulation of Innate Immune Response Caused by Viruses. Front. Immunol. 11, 558341. doi:10.3389/fimmu.2020.558341

Imai, Y., Kuba, K., Neely, G. G., Yaghubian-Malhami, R., Perkmann, T., van Loo, G., et al. (2008). Identification of Oxidative Stress and Toll-like Receptor 4 Signaling as a Key Pathway of Acute Lung Injury. Cell 133 (2), 235-249. doi:10.1016/j.cell.2008.02.043

Jackson, W. T. (2015). Viruses and the Autophagy Pathway. Virology 479-480, 450-456. doi:10.1016/j.virol.2015.03.042

Joubert, P.-E., Meiffren, G., Grégoire, I. P., Pontini, G., Richetta, C., Flacher, M., et al. (2009). Autophagy Induction by the Pathogen Receptor CD46. Cell Host \& Microbe 6 (4), 354-366. doi:10.1016/j.chom.2009.09.006

Kang, R., Zeh, H. J., Lotze, M. T., and Tang, D. (2011). The Beclin 1 Network Regulates Autophagy and Apoptosis. Cell Death Differ 18 (4), 571-580. doi:10.1038/cdd.2010.191

Kawai, T., and Akira, S. (2010). The Role of Pattern-Recognition Receptors in Innate Immunity: Update on Toll-like Receptors. Nat. Immunol. 11 (5), 373-384. doi:10.1038/ni.1863

Kim, H. J., Lee, S., and Jung, J. U. (2010). When Autophagy Meets Viruses: a Double-Edged Sword with Functions in Defense and Offense. Semin. Immunopathol 32 (4), 323-341. doi:10.1007/s00281-010-0226-8

Kim, N., Kim, M.-J., Sung, P. S., Bae, Y. C., Shin, E.-C., and Yoo, J.-Y. (2016). Interferon-inducible Protein SCOTIN Interferes with HCV Replication through the Autolysosomal Degradation of NS5A. Nat. Commun. 7, 10631. doi:10.1038/ncomms 10631

Kumar, J., Jain, S., Meena, J., and Yadav, A. (2021). Efficacy and Safety of Hydroxychloroquine/chloroquine against SARS-CoV-2 Infection: A Systematic Review and Meta-Analysis. J. Infect. Chemother. 27 (6), 882-889. doi:10.1016/j.jiac.2021.02.021

Kyei, G. B., Dinkins, C., Davis, A. S., Roberts, E., Singh, S. B., Dong, C., et al. (2009). Autophagy Pathway Intersects with HIV-1 Biosynthesis and Regulates Viral Yields in Macrophages. J. Cel Biol 186 (2), 255-268. doi:10.1083/jcb.200903070

Lee, M. S., and Kim, Y.-J. (2007). Signaling Pathways Downstream of PatternRecognition Receptors and Their Cross Talk. Annu. Rev. Biochem. 76, 447-480. doi:10.1146/annurev.biochem.76.060605.122847

Lee, N.-R., Ban, J., Lee, N.-J., Yi, C.-M., Choi, J.-Y., Kim, H., et al. (2018). Activation of RIG-I-Mediated Antiviral Signaling Triggers Autophagy through the MAVSTRAF6-Beclin-1 Signaling Axis. Front. Immunol. 9, 2096. doi:10.3389/ fimmu.2018.02096
Lennemann, N. J., and Coyne, C. B. (2015). Catch Me if You Can: the Link between Autophagy and Viruses. Plos Pathog. 11 (3), e1004685. doi:10.1371/ journal.ppat.1004685

Levine, B., and Kroemer, G. (2019). Biological Functions of Autophagy Genes: A Disease Perspective. Cell 176 (1-2), 11-42. doi:10.1016/ j.cell.2018.09.048

Levine, B. (2005). Eating Oneself and Uninvited GuestsAutophagy-Related Pathways in Cellular Defense. Cell 120 (2), 159-162. doi:10.1016/s00928674(05)00043-7

Levine, B., and Kroemer, G. (2008). Autophagy in the Pathogenesis of Disease. Cell 132 (1), 27-42. doi:10.1016/j.cell.2007.12.018

Levine, B., Mizushima, N., and Virgin, H. W. (2011). Autophagy in Immunity and Inflammation. Nature 469 (7330), 323-335. doi:10.1038/nature09782

Leymarie, O., Lepont, L., and Berlioz-Torrent, C. (2017). Canonical and Noncanonical Autophagy in HIV-1 Replication Cycle. Viruses 9 (10). doi:10.3390/ v9100270

Li, J., Liu, Y., Wang, Z., Liu, K., Wang, Y., Liu, J., et al. (2011). Subversion of Cellular Autophagy Machinery by Hepatitis B Virus for Viral Envelopment. J. Virol. 85 (13), 6319-6333. doi:10.1128/jvi.02627-10

Li, X., Li, Y., Fang, S., Su, J., Jiang, J., Liang, B., et al. (2017). Downregulation of Autophagy-Related Gene ATG5 and GABARAP Expression by IFN- $\Lambda 1$ Contributes to its Anti-HCV Activity in Human Hepatoma Cells. Antiviral Res. 140, 83-94. doi:10.1016/j.antiviral.2017.01.016

Liang, Q., Seo, G. J., Choi, Y. J., Kwak, M.-J., Ge, J., Rodgers, M. A., et al. (2014). Crosstalk between the cGAS DNA Sensor and Beclin-1 Autophagy Protein Shapes Innate Antimicrobial Immune Responses. Cell Host \& Microbe 15 (2), 228-238. doi:10.1016/j.chom.2014.01.009

Loi, M., Müller, A., Steinbach, K., Niven, J., Barreira da Silva, R., Paul, P., et al. (2016). Macroautophagy Proteins Control MHC Class I Levels on Dendritic Cells and Shape Anti-viral CD8 + T Cell Responses. Cel Rep. 15 (5), 1076-1087. doi:10.1016/j.celrep.2016.04.002

Londino, J. D., Lazrak, A., Noah, J. W., Aggarwal, S., Bali, V., Woodworth, B. A., et al. (2015). Influenza Virus M2 Targets Cystic Fibrosis Transmembrane Conductance Regulator for Lysosomal Degradation during Viral Infection. FASEB j. 29 (7), 2712-2725. doi:10.1096/fj.14-268755

Lünemann, J. D., and Münz, C. (2009). Autophagy in CD4+ T-Cell Immunity and Tolerance. Cel Death Differ 16 (1), 79-86. doi:10.1038/cdd.2008.113

Mancias, J. D., and Kimmelman, A. C. (2016). Mechanisms of Selective Autophagy in Normal Physiology and Cancer. J. Mol. Biol. 428 (9), 1659-1680. doi:10.1016/ j.jmb.2016.02.027

McKnight, N. C., and Yue, Z. (2013). Beclin 1, an Essential Component and Master Regulator of PI3K-III in Health and Disease. Curr. Pathobiol Rep. 1 (4), 231-238. doi:10.1007/s40139-013-0028-5

Miao, G., Zhao, H., Li, Y., Ji, M., Chen, Y., Shi, Y., et al. (2021). ORF3a of the COVID-19 Virus SARS-CoV-2 Blocks HOPS Complex-Mediated Assembly of the SNARE Complex Required for Autolysosome Formation. Dev. Cel 56 (4), 427-442. doi:10.1016/j.devcel.2020.12.010

Mizushima, N. (2018). A Brief History of Autophagy from Cell Biology to Physiology and Disease. Nat. Cel Biol 20 (5), 521-527. doi:10.1038/s41556018-0092-5

Mizushima, N., and Levine, B. (2020). Autophagy in Human Diseases. N. Engl. J. Med. 383 (16), 1564-1576. doi:10.1056/nejmra2022774

Negishi, H., Taniguchi, T., and Yanai, H. (2018). The Interferon (IFN) Class of Cytokines and the IFN Regulatory Factor (IRF) Transcription Factor Family. Cold Spring Harb Perspect. Biol. 10 (11), a028423. doi:10.1101/ cshperspect.a028423

Ni, G., Ma, Z., Wong, J. P., Zhang, Z., Cousins, E., Major, M. B., et al. (2020). PPP6C Negatively Regulates STING-dependent Innate Immune Responses. mBio 11 (4), e01728. doi:10.1128/mBio.01728-20

Oku, M., and Sakai, Y. (2018). Three Distinct Types of Microautophagy Based on Membrane Dynamics and Molecular Machineries. Bioessays 40 (6), e1800008. doi:10.1002/bies.201800008

Padman, B. S., Nguyen, T. N., and Lazarou, M. (2017). Autophagosome Formation and Cargo Sequestration in the Absence of LC3/GABARAPs. Autophagy 13 (4), 772-774. doi:10.1080/15548627.2017.1281492

Perot, B. P., Ingersoll, M. A., and Albert, M. L. (2013). The Impact of Macroautophagy on CD8+T-Cell-Mediated Antiviral Immunity. Immunol. Rev. 255 (1), 40-56. doi:10.1111/imr.12096 
Pleet, M. L., Branscome, H., DeMarino, C., Pinto, D. O., Zadeh, M. A., Rodriguez, M., et al. (2018). Autophagy, EVs, and Infections: A Perfect Question for a Perfect Time. Front. Cel. Infect. Microbiol. 8, 362. doi:10.3389/fcimb.2018.00362

Puleston, D. J., Zhang, H., Powell, T. J., Lipina, E., Sims, S., Panse, I., et al. (2014). Autophagy Is a Critical Regulator of Memory CD8(+) T Cell Formation. Elife 3, e03706. doi:10.7554/eLife.03706

Richetta, C., Grégoire, I. P., Verlhac, P., Azocar, O., Baguet, J., Flacher, M., et al. (2013). Sustained Autophagy Contributes to Measles Virus Infectivity. Plos Pathog. 9 (9), e1003599. doi:10.1371/journal.ppat.1003599

Rubinsztein, D. C., Mariño, G., and Kroemer, G. (2011). Autophagy and Aging. Cell 146 (5), 682-695. doi:10.1016/j.cell.2011.07.030

Saha, S., Panigrahi, D. P., Patil, S., and Bhutia, S. K. (2018). Autophagy in Health and Disease: A Comprehensive Review. Biomed. Pharmacother. 104, 485-495. doi:10.1016/j.biopha.2018.05.007

Shojaei, S., Suresh, M., Klionsky, D. J., Labouta, H. I., and Ghavami, S. (2020). Autophagy and SARS-CoV-2 Infection: A Possible Smart Targeting of the Autophagy Pathway. Virulence 11 (1), 805-810. doi:10.1080/ 21505594.2020.1780088

Shrivastava, S., Raychoudhuri, A., Steele, R., Ray, R., and Ray, R. B. (2011). Knockdown of Autophagy Enhances the Innate Immune Response in Hepatitis C Virus-Infected Hepatocytes. Hepatology 53 (2), 406-414. doi:10.1002/hep. 24073

Singh, B., Ryan, H., Kredo, T., Chaplin, M., and Fletcher, T. (2021). Chloroquine or Hydroxychloroquine for Prevention and Treatment of COVID-19. Cochrane Database Syst. Rev. 2 (2), Cd013587. doi:10.1002/14651858.CD013587.pub2

Sir, D., and Ou, J.-h. J. (2010). Autophagy in Viral Replication and Pathogenesis. Mol. Cell 29 (1), 1-7. doi:10.1007/s10059-010-0014-2

Song, J., Hu, Y., Li, J., Zheng, H., Wang, J., Guo, L., et al. (2018). Suppression of the Toll-like Receptor 7-dependent Type I Interferon Production Pathway by Autophagy Resulting from Enterovirus 71 and Coxsackievirus A16 Infections Facilitates Their Replication. Arch. Virol. 163 (1), 135-144. doi:10.1007/s00705-017-3592-x

Stanifer, M. L., Pervolaraki, K., and Boulant, S. (2019). Differential Regulation of Type I and Type III Interferon Signaling. Int. J. Mol. Sci. 20 (6), 1445. doi:10.3390/ijms20061445

Stephenson, L. M., Miller, B. C., Ng, A., Eisenberg, J., Zhao, Z., Cadwell, K., et al. (2009). Identification ofAtg5-dependent Transcriptional Changes and Increases in Mitochondrial Mass inAtg5-Deficient $\mathrm{T}$ Lymphocytes. Autophagy 5 (5), 625-635. doi:10.4161/auto.5.5.8133

Tanaka, A., Jin, Y., Lee, S.-J., Zhang, M., Kim, H. P., Stolz, D. B., et al. (2012). Hyperoxia-induced LC3B Interacts with the Fas Apoptotic Pathway in Epithelial Cell Death. Am. J. Respir. Cel Mol Biol 46 (4), 507-514. doi:10.1165/rcmb.2009-0415oc
Tang, H., Da, L., Mao, Y., Li, Y., Li, D., Xu, Z., et al. (2009). Hepatitis B Virus X Protein Sensitizes Cells to Starvation-Induced Autophagy via Up-Regulation of Beclin 1 Expression. Hepatology 49 (1), 60-71. doi:10.1002/hep.22581

Tey, S.-K., and Khanna, R. (2012). Host Immune System Strikes Back. Autophagy 8 (12), 1839-1841. doi:10.4161/auto.21860

Wei, Y., Liu, M. X., Li, X. X., Liu, J. G., and Li, H. (2018). Origin of the Autophagosome Membrane in Mammals. Biomed. Res. Int. 2018, 1012789. doi:10.1155/2018/1012789

Weston, S., Coleman, C. M., Haupt, R., Logue, J., Matthews, K., Li, Y., et al. (2020). Broad Anti-coronavirus Activity of Food and Drug Administration-Approved Drugs against SARS-CoV-2 In Vitro and SARS-CoV In Vivo. J. Virol. 94 (21), e01218. doi:10.1128/JVI.01218-20

Xu, X.-x., Wan, H., Nie, L., Shao, T., Xiang, L.-X., and Shao, J.-z. (2018). RIG-I: a Multifunctional Protein beyond a Pattern Recognition Receptor. Protein Cell 9 (3), 246-253. doi:10.1007/s13238-017-0431-5

Xu, X., Araki, K., Li, S., Han, J.-H., Ye, L., Tan, W. G., et al. (2014). Autophagy Is Essential for Effector CD8+ T Cell Survival and Memory Formation. Nat. Immunol. 15 (12), 1152-1161. doi:10.1038/ni.3025

Yang, Y., and Klionsky, D. J. (2020). Autophagy and Disease: Unanswered Questions. Cel Death Differ 27 (3), 858-871. doi:10.1038/s41418-019-0480-9

Zachari, M., and Ganley, I. G. (2017). The Mammalian ULK1 Complex and Autophagy Initiation. Essays Biochem. 61 (6), 585-596. doi:10.1042/ ebc20170021

Zalckvar, E., Berissi, H., Eisenstein, M., and Kimchi, A. (2009). Phosphorylation of Beclin 1 by DAP-Kinase Promotes Autophagy by Weakening its Interactions with Bcl-2 and Bcl-XL. Autophagy 5 (5), 720-722. doi:10.4161/auto.5.5.8625

Conflict of Interest: The authors declare that the research was conducted in the absence of any commercial or financial relationships that could be construed as a potential conflict of interest.

Publisher's Note: All claims expressed in this article are solely those of the authors and do not necessarily represent those of their affiliated organizations, or those of the publisher, the editors and the reviewers. Any product that may be evaluated in this article, or claim that may be made by its manufacturer, is not guaranteed or endorsed by the publisher.

Copyright (c) 2021 Liang, Liu, He, Ai, Meng, Zhang, Yu, Wang and Liu. This is an open-access article distributed under the terms of the Creative Commons Attribution License (CC BY). The use, distribution or reproduction in other forums is permitted, provided the original author(s) and the copyright owner(s) are credited and that the original publication in this journal is cited, in accordance with accepted academic practice. No use, distribution or reproduction is permitted which does not comply with these terms. 